\title{
Investigating the Effect of Expiratory Time Constant on Outcome in Intubated Patients with Acute Respiratory Failure Caused by COVID-19 in Critical Care Unit: A Research Study
}

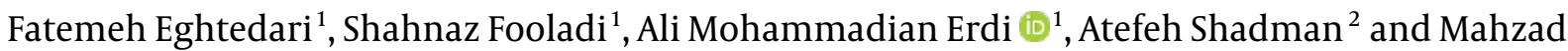 \\ Yousefian (iD 1 , * \\ ${ }^{1}$ Department of Anesthesiology, School of Medicine, Adabil University of Medical Scienes, Ardabil, Iran \\ ${ }^{2}$ Determinations of Health Research Center, School of Medicine, Adabil University of Medical Scienes, Ardabil, Iran \\ "Corresponding author: Department of Anesthesiology, School of Medicine, Adabil University of Medical Scienes, Ardabil, Iran. Email: dr_mahzad@yahoo.com
}

Received 2021 September 18; Revised 2021 November 23; Accepted 2021 November 27.

\begin{abstract}
Background: The coronavirus disease 2019 (COVID-19) has a high prevalence and mortality worldwide. Thousands of patients with acute respiratory failure caused by COVID-19 are daily hospitalized in intensive care units (ICUs) around the world. Many of these patients require full mechanical respiratory support and long-term ventilator use. Using different ventilators and calculating important variables can be helpful in meeting therapeutic needs of patients.

Objectives: The aim of present study was to investigate the effect of expiratory time constant (RCEXP) on the course of treatment and duration of mechanical ventilation in patients with acute respiratory failure hospitalized in ICU.

Methods: The present cross-sectional study was conducted on 60 patients with acute respiratory failure who were hospitalized in the ICU and underwent mechanical ventilation due to COVID-19 in the first six months of 2020. The variables of RCEXP, lung compliance and lung resistance in all patients were recorded daily and analyzed. Then, based on clinical outcome, the patients were divided into two groups: the patients with wean outcome $(\mathrm{N}=40)$ and those with death outcome $(\mathrm{N}=20)$.

Results: The mean \pm SD of lung compliance in patients who were separated from ventilator and patients with death outcome were $74.73(18.58) \mathrm{mL} / \mathrm{cm} \mathrm{H}_{2} \mathrm{O}$ and $36.92(10.56) \mathrm{mL} / \mathrm{cm} \mathrm{H}_{2} \mathrm{O}$, respectively, which was statistically significant $(\mathrm{P}=0.001)$. The mean $\pm \mathrm{SD}$ of lung resistance in patients who were separated from ventilator and patients with death outcome were calculated at 9.25 (4.62) and 14 (6.5), respectively, which was statistically significant $(P=0.015)$. Also, there was a statistically significant difference between the two groups in terms of mean $\pm \operatorname{SD}$ of $\operatorname{RCEXP}(0.67(0.23)$ vs. $0.49(0.19), \mathrm{P}=0.010)$.

Conclusions: According to the results of this study, there was a significant difference between high resistance, low compliance, RCEXP, and weaning success of intubation in patients hospitalized in the ICU.
\end{abstract}

Keywords: Compliance, Resistance, RCEXP, Mechanical Ventilation

\section{Background}

The coronavirus disease 2019 (COVID-19), as an epidemic disease that might lead to death, has become a major global concern (1). Human life has been impressed by the advent of this disease, both personally and professionally. e.g., in critical care of residents and physicians' lives, compared to the previous coronavirus epidemics of severe acute respiratory syndrome (SARS) and the Middle East respiratory syndrome (MERS)) $(2,3)$. The virus is rapidly spread through close contact, often via small droplets produced by coughing, sneezing, or talking. Due to the examinations and close contact of the physicians and health care workers with the head and neck region and airways, they are particularly at high risk of infection from aerosol and droplet contamination (4). However, about $15-20 \%$ of the cases are believed to be severe, and many cases are asymptomatic (5). Hemodynamic changes are not significant with normal mechanical ventilation via spontaneous respiration. However, the patient's hemodynamics are greatly affected in several pathological conditions, such as hypovolemia, asthmatic status, pneumothorax, and respiration (6).

Despite the beneficial effects of mechanical ventilation on the pathophysiology of acute pulmonary insufficiency, it is associated with complications such as increased risk of sinusitis, airway injury, thromboembolism, gastrointestinal bleeding, pneumonia, ventilator dependence, pul- 
monary barotrauma, and lung injury caused (7). As the need for mechanical respiration ends, the patient should be separated from the ventilator as soon as possible (8). Due to shortage of beds in the intensive care units (ICUs) and lack of mechanical ventilation equipment in hospitals, finding the methods to shorten hospitalization time and reduce ventilator dependence can help reduce the cost of treatment, as well as the complications of mechanical ventilation (9). Hence, timely, rapid, uncomplicated, and successful separation by shortening the mechanical ventilation period reduces ventilation complications (e.g., decreased cardiac output and infections caused by artificial ventilation), hyperventilation and hypoventilation, atelectasis, oxygen poisoning, barotrauma, and psychological ventilator dependence. Furthermore, unnecessary prolonged mechanical ventilation is associated with increased mortality (10-12).

Hypoxia is often the main cause of critically ill patients who need to be transferred to the ICU (13), acute diffuse lung injury, inflammatory lung injury leading to increased pulmonary vascular permeability, increased lung weight, and lung tissue loss (14).

Separating the patient from the ventilator merely based on clinical criteria is usually an incorrect issue which leads to early and unsuccessful separation (15). Furthermore, physiological tests should be considered as a predictor of separation results.

Separation often fails due to the existence of several influential factors. One of the reasons for failure in separation is the existence of several influential factors. Thus, the indicators and criteria that consider a pathophysiological mechanism are not valid and accurate enough. Meanwhile, the indicators that consider multiple mechanisms have higher accuracy (16). There is a higher tendency to determine specific, measurable indicators to predict separation outcomes. However, a single indicator that provides successful outcomes in this prediction has not been identified yet (8).

Expiratory time constant (RCEXP) enables us to assess the respiratory mechanics. The RCEXP less than 0.5 seconds indicates a decrease in lung compliance. In acute respiratory distress syndrome (ARDS) patients, the RCEXP is usually 0.4 to 0.6 seconds, and in individuals with more severe ARDS, this value is shorter, indicating the low compliance of lung. In patients with pulmonary fibrosis or chest wall stiffness, such as kyphoscoliosis, RCEXP is usually very short, ranging from 0.15 to 0.25 seconds. RCEXP more than 0.7 seconds indicates an increase in lung resistance, which may be associated with increased compliance in chronic obstructive pulmonary disease (COPD) patients with pulmonary emphysema. Prolonged RCEXP is common in patients with COPD and asthma. For example, in patients with severe bronchospasm, RCEXP can be up to 3 seconds. If the patient does not have COPD or asthma, prolonged RCEXP may indicate incorrect position or endotracheal contraction (17). There is a wide variety of factors for determining the weaning process time and extubating. Although RCEXP can be used as a factor for successful prediction of weaning, currently there is no study on determining the weaning process time. Considering the importance of predicting successful weaning time in patients with COVID-19 and untimely separation of patients, in this study, we surveyed the effects of these factors on weaning time.

\section{Objectives}

We aimed to investigate the effect of expiratory time constant on the course of treatment and duration of mechanical ventilation in patients with acute respiratory failure caused by COVID-19 in ICU.

\section{Methods}

\subsection{Patients and Data Collection}

The present cross-sectional research was conducted on 60 patients hospitalized in the ICU of Imam Khomeini Hospital of Ardabil University of Medical Sciences, Iran, due to respiratory failure caused by COVID-19, who underwent mechanical ventilation in the first six months of 2020. The variables of RCEXP, lung compliance and lung resistance in all patients were recorded daily and analyzed. Then, based on clinical outcome, the patients were divided into two groups: the patients with wean outcome $(\mathrm{N}=40)$ and those with death outcome $(\mathrm{N}=20)$. The exclusion criteria were hospitalization due to non-pulmonary reasons and hospitalization time less than 48 hours. Data were collected using a checklist, including demographic information extracted from the patients' hospital records. All patients who were hospitalized and intubated due to pulmonary problems and underwent ventilator for more than 48 hours were included. The spontaneous awakening trial (SAT) and spontaneous breathing trial(SBT) protocols were used to separate patients from the ventilator. The following formula was used to calculate lung compliance:

Lung compliance $(C)=$ Changes in lung volume (V)/Change in transpulmonary pressure [Alvoelar pressure (Palv)-Pleural pressure (Ppl)] (18).

Also, body-plethysmography has been demonstrated to calculate the airway resistance (Raw) as a ratio of driving alveolar pressure to airflow (19). RCEXP is a mechanical respiratory measurement using Hamilton ventilators. Since RCEXP is a product of lung capacity and resistance, this variable enables us to assess respiratory mechanics. 
It is very useful for diagnosing lung disease and its severity, ventilator settings, monitoring of susceptible position, and understanding some respiratory events. In a patient with normal ventilation and normal lungs, the normal RCEXP is between 0.5 and 0.7 seconds. However, it is important to check that the lung capacity and resistance values are in the normal range. Since lung disease reduces lung capacity and increases lung resistance, it may lead to false normalization of RCEXP (17).

\subsection{Ethical Statement}

This study was approved by the Ethics Committee of Ardabil University of Medical Sciences, Iran (code: IR.ARUMS. RE1398.322).

\subsection{Statistical Analysis}

After recording the data and completing the checklist containing such information as lung compliance, lung resistance, RCEXP and age, gender, and underlying disease, statistical analysis was performed using SPSS software version 16 . The mean \pm SD were reported for quantitative variables, and percentage and frequency were reported for qualitative variables. The t-test and chi-square test were used to compare the two groups. P-value less than 5\% was considered as statistically significant.

\section{Results}

The present study was conducted on 60 patients (age range: 15 - 90 years) referred to Imam Khomeini Hospital in Ardabil, Iran due to COVID-19 disease. According to the results, 40 (66.7\%) patients were separated from the ventilator, and 20 (33.3\%) patients died. The mean age of participants was 54.98 years, with a standard deviation of 19.74 . The mean age and standard deviation in patients who were separated from the ventilator and those who had death outcome were $48.48 \pm 18.24$ and $68 \pm 16.12$ years, respectively, which was statistically significant $(\mathrm{P}=0.001)$ (Table 1).

In the present study, 32 (53.3\%) participants were male, and 28 (46.7\%) were female. The outcome between the two genders did not show a statistically significant difference. Statistical tests showed that out of 40 patients, 18 cases had a history of underlying disease. This difference between the two groups was not statistically significant $(\mathrm{P}=0.714)$.

The mean \pm SD of lung compliance in patients who were separated from ventilator and patients with death outcome were 74.73 (18.58) and 36.92 (10.56), respectively, which was statistically significant $(\mathrm{P}=0.001)$. Also, the mean \pm SD of lung resistance in patients who were separated from ventilator and those with death outcome were calculated at 9.25 (4.62) and 14 (6.5), respectively, which was statistically significant $(\mathrm{P}=0.001)$. There was a statistically significant difference between the two groups in terms of $\operatorname{RCEXP}(P=0.010)$ (Table 2). Finally, there was an inverse relationship between high resistance, low compliance, and the RCEXP levels and the result of successful weaning.

\section{Discussion}

In the present study, we investigated the effect of expiratory time constant on the course of treatment and duration of mechanical ventilation in COVID-19 patients admitted to the ICU. According to the results, $66.7 \%$ of the patients were separated from mechanical ventilation system, and $33.3 \%$ of them died. There was a statistically significant difference in the age of patients between the two groups. Also, 53.3\% of the participants were male, and $46.7 \%$ were female, and the outcome between the two genders did not show a statistically significant difference. In a similar study conducted on clinical features and prognosis of invasive ventilation in patients who were hospitalized with COVID-19, a large number of critically ill patients admitted to ICU were older males with poor outcomes and a high mortality rate (20). Furthermore, a very high mortality rate of critically ill patients with COVID-19 was reported; since these patients had dyspnea and required mechanical ventilation, they were at a higher risk for death (21). The results of an observational study evaluating the effects of body mass index (BMI) on the mortality of critically ill patients demonstrated that regardless of age and gender, BMI could increase the risk of mortality (22). According to Mahmoodpoor et al., almost $60 \%$ of the patients underwent mechanical ventilation, $25 \%$ underwent non-invasive ventilation, and $15 \%$ received supplementary oxygen through facial oxygen masks (23).

In our study, $45 \%$ of patients had a history of underlying disease. In patients who underwent mechanical ventilation with normal lungs, the normal RCEXP was between 0.5 and 0.7 seconds. It is important to check that lung capacity and resistance values are within the normal range. Since lung disease decreases lung capacity and increases lung resistance, and also measuring lung volumes and airway resistance is often important to provide an adequate characterization of the pattern of lung disease (24), it may lead to false normalization of RCEXP. To the best of our knowledge, based on the available data within the first hours of hospitalization, predicting the need for mechanical ventilation, a risk score has been developed that should be validated to determine its further applicability in other populations (25).

Furthermore, controlled modes in COVID-19 (26) and positive end-expiratory pressure (PEEP) application are 


\begin{tabular}{|c|c|c|c|c|}
\hline Variable & Total $(N=40)$ & Weaning $(\mathrm{N}=40)$ & Death $(N=20)$ & P-Value \\
\hline Age & $120 \pm 30$ & $48.48 \pm 18.24$ & $68 \pm 16.12$ & 0.01 \\
\hline Gender & & & & 0.714 \\
\hline Male & $32(5.3)$ & $22(68.8)$ & $10(31.3)$ & \\
\hline Female & $28(46.7)$ & $18(64.3)$ & $10(35.7)$ & \\
\hline Respiratory disease & & & & 0.714 \\
\hline Yes & $17(28.3)$ & $18(64.3)$ & $10(35.7)$ & \\
\hline No & $43(71.7)$ & $22(68.8)$ & $10(31.2)$ & \\
\hline Underlying disease & & & & 0.714 \\
\hline Yes & $28(46.7)$ & $5(29.4)$ & $12(70.6)$ & \\
\hline No & $32(3.53)$ & $35(81 / 4)$ & $8(18 / 6)$ & \\
\hline
\end{tabular}

\begin{tabular}{lccc}
\hline \multicolumn{4}{l}{ Table 2. The Mean \pm SD of the Resistance, Compliance, and RCEXP ${ }^{\mathrm{a}}$} \\
\hline Variable & Weaning & Death & P-Value \\
\hline Resistance & $9.25 \pm 4.62$ & $14 \pm 6.5$ & 0.015 \\
Compliance & $74.73 \pm 18.58$ & $36.92 \pm 10.56$ & 0.001 \\
RCEXP & $0.67 \pm 0.23$ & $0.47 \pm 0.19$ & 0.01 \\
\hline
\end{tabular}

${ }^{\mathrm{a}}$ Values are expressed as mean $\pm \mathrm{SD}$.

linked to improved arterial blood gas in patients undergoing gynecologic laparoscopy as one of the strategies for improving the respiratory status (27). A previous case report showed that paying attention to happy hypoxemia critically improved the health status of COVID-19 patients (28). Also, dexamethasone was associated with reduced need for mechanical ventilation as observed in another study, by improving compliance and promoting better oxygenation (29). According to the results of a study by Asri et al., dexmedetomidine may improve arterial oxygenation during one-lung ventilation (OLV) in adult patients under the thoracic surgery, and can be a suitable anesthetic factor for thoracic surgery (30). In a previous study, we demonstrated the effect of vitamin D supplements on expediting the weaning process in patients with the stroke (31). Another study found a significant correlation between total and ionized calcium, but this correlation was not significant between corrected and ionized calcium. They proposed hypocalcemia as a predictor of disease severity and mortality (32).

RCEXP less than 0.5 seconds indicates a decrease in lung compliance. In ARDS patients, the RCEXP is usually in the range of 0.4 to 0.6 seconds. In patients with more severe ARDS, it is shorter, indicating low compliance. In patients with pulmonary fibrosis or chest wall stiffness such as kyphoscoliosis, RCEXP is usually very short, rang- ing from 0.15 to 0.25 seconds. RCEXP more than 0.7 seconds indicates an increase in lung resistance, which may be associated with increased compliance in COPD patients with pulmonary emphysema (17). Prolonged RCEXP is common in patients with COPD and asthma. In patients with severe bronchospasm, RCEXP can be up to 3 seconds. If the patient does not have COPD or asthma, prolonged RCEXP may indicate incorrect position or endotracheal contraction (17). In patients with normal lungs under mechanical ventilation, normal RCEXP is in the range of 0.5 and 0.7 seconds. In ARDS patients, RCEXP is in the range of 0.4 to 0.6 seconds. RCEXP less than 0.5 seconds indicates decreased lung compliance. RCEXP longer than 0.7 seconds indicates increased lung resistance and increased lung compliance, as seen in patients with COPD and asthma.

In this study, we found a significant difference in lung compliance in the patients who were separated from mechanical ventilation compared to those who died. This finding is in line with the results of a recent study conducted on 113 patients with success and non-success extubation groups, indicating successful extubation in $13.1 \%$ of patients (33). In another study, P. Candik examined the relationship between the expiratory time constant and ventilator separation parameters in patients hospitalized with respiratory failure. The results showed that RCEXP was associated with ventilator separation and extubation, so that RCEXP was an important parameter for extubation (34).

In the present study, a statistically significant difference was found between the two groups in terms of mean and standard deviation of RCEXP. Thus, our results confirm these findings, indicating a significant difference in RCEXP between the two experimental groups. Okabe et al. reported that lung-thorax compliance is a potential indicator for extubation failure in patients admitted to the ICU, suggesting that lung-thorax compliance measure- 
ment can be a good index for extubation failure in the ICU. They also indicated that measurement of this index during a spontaneous breathing trial potentially can be an indicator of extubation failure in postoperative patients (33).

Basiri et al (35). conducted a study on comparison of pressure index (CROP) and rapid shallow breathing index (RSBI) separation indices in predicting the outcome of mechanical ventilation in 80 patients admitted to the ICU. Based on their results, the sensitivity of CROP and RSBI indices was $85 \%$ and $98.2 \%$, respectively, and their specificity was $5 \%$ and $26 \%$, respectively. The diagnostic accuracy of CROP (85.2\%) was higher than that of RSBI index. In this study, CROP (compliance) had the highest diagnostic accuracy with high sensitivity and specificity. According to the requirement of endotracheal intubation and mechanical ventilation in ARDS in COVID-19 patients, some factors, including severe respiratory distress, loss of consciousness, and hypoxia, were the most important reasons for intubation (36).

Al-Rawas et al. evaluated RCEXP as a criterion for determining respiratory system compliance and resistance (37). In this study, 92 patients with acute respiratory failure who underwent mechanical ventilation through different ventilator modes were evaluated. This study concluded that the RCEXP is a good criterion for determining the respiratory system compliance and resistance (37).

We also witnessed a significant difference between successful and unsuccessful extubation groups. This supports the findings by Okabe et al., in which 162 (93.6\%) patients were successfully extubated, and 11 (6.4\%) patients experienced unsuccessful extubation. The mean lung compliance in the successful and unsuccessful extubation groups was $71.9 \pm 0.23$ and $43.3 \pm 14.6$, respectively, which was a statistically significant difference $(\mathrm{P}<0.0001)$ (33).

Our results support the use of expiratory time constant on the course of treatment and duration of mechanical ventilation in patients with acute respiratory failure in the ICU. The main limitations of this study included a singlecenter nature of the study and a small sample size.

\subsection{Conclusions}

Since RCEXP was lower in the group with death outcome and lower level of this criterion is more common in restrictive lung diseases, it can be concluded that most severe lung diseases caused by COVID-19 have ARDS, and there is a significant relationship between low RCEXP and mortality rate. Further studies with larger sample sizes are required to confirm the results.

\section{Footnotes}

Authors' Contribution: MY and FE., Study design, Data analysis, Writing - original draft, Writing - review \& editing. ASH Data analysis FE. and AMA, Data collection. The author(s) read and approved the final manuscript.

Conflict of Interests: The authors declare no conflict of interest.

Ethical Approval: This study was approved by the Ethics Committee of Ardabil University of Medical Sciences (code: IR.ARUMS. RE1398.322).

Funding/Support: None.

Informed Consent: It was not declared by the author.

\section{References}

1. Dastan F, Abedini A, Shahabi S, Kiani A, Saffaei A, Zare A. Sitagliptin Repositioning in SARS-CoV-2: Effects on ACE-2, CD-26, and Inflammatory Cytokine Storms in the Lung. Iran J Allergy Asthma Immunol. 2020;19(S1):10-2. doi: 10.18502/ijaai.v19i(s1.r1).2849. [PubMed: 32534505].

2. Aisa I, Llau J, Gonzalez JM, Delgado C, Otero M, Romero CS, et al. Impact of COVID-19 Pandemic on Anesthesia and Critical Care Residents in Spain. Anesth Pain Med. 2021;11(4). e116836. doi: 10.5812/aapm.116836. [PubMed: 34692440]. [PubMed Central: PMC8520680].

3. Ali H, Ismail AA, Abdalwahab A. Mental Stress in Anesthesia and Intensive Care Physicians During COVID-19 Outbreak. Anesth Pain Med. 2020;10(5). e106623. doi: 10.5812/aapm.106623. [PubMed: 34150567]. [PubMed Central: PMC8207887].

4. Hassani V, Amniati S, Ahmadi A, Mohseni M, Sehat-Kashani S, Nikoubakht N, et al. Emergency Tracheostomy in Two Airway Trauma Patients Suspected of COVID-19: A Case Report. Anesth Pain Med. 2020;10(4). e104648. doi: 10.5812/aapm.104648. [PubMed: 33134149]. [PubMed Central: PMC7539045].

5. Pergolizzi JJ, Magnusson P, LeQuang JA, Breve F, Paladini A, Rekatsina $\mathrm{M}$, et al. The Current Clinically Relevant Findings on COVID-19 Pandemic. Anesth Pain Med. 2020;10(2). e103819. doi: 10.5812/aapm.103819. [PubMed: 32754437]. [PubMed Central: PMC7352949].

6. Bilehjani E, Nader N, Farzin H, Haghighate Azari M, Fakhari S. The Evaluation of Factors Affecting Hemodynamic Variability in Mechanically-Ventilated Patients After Cardiac Surgery. Anesth Pain Med. 2020;10(4). e101832. doi: 10.5812/aapm.101832. [PubMed: 33134143]. [PubMed Central: PMC7539053].

7. McLean SE, Jensen LA, Schroeder DG, Gibney NR, Skjodt NM. Improving adherence to a mechanical ventilation weaning protocol for critically ill adults: outcomes after an implementation program. Am J Crit Care. 2006;15(3):299-309. [PubMed:16632772].

8. Alvisi R, Volta CA, Righini ER, Capuzzo M, Ragazzi R, Verri M, et al. Predictors of weaning outcome in chronic obstructive pulmonary disease patients. Eur Respir J. 2000;15(4):656-62. doi: 10.1034/j.13993003.2000.15d06.x. [PubMed: 10780755].

9. Madani SJ, Saghafinia M, Sedighi Nezhad H, Ebadi A, Ghochani A, Fazel Tavasoli A, et al. Validity of Integrative Weaning Index of Discontinuation From Mechanical Ventilation in Iranian ICUs. Thrita. 2013;2(2):628. doi: $10.5812 /$ thrita.12827.

10. Delisle S, Francoeur M, Albert M, Ouellet P, Bellemare P, Arsenault P. Preliminary evaluation of a new index to predict the outcome of a spontaneous breathing trial. Respir Care. 2011;56(10):1500-5. doi: 10.4187/respcare.00768. [PubMed: 21310112]. 
11. Sellares J, Ferrer M, Torres A. Predictors of weaning after acute respiratory failure. Minerva Anestesiol. 2012;78(9):1046-53. [PubMed: 22743787].

12. Jalalian H, Aslani J, Panahi Y. [Factors affecting the duration of mechanical ventilation device isolation of patients in intensive care units]. Kowsar Med J. 2009;14(3):163-8. Persian.

13. Ards Definition Task Force, Ranieri VM, Rubenfeld GD, Thompson BT, Ferguson ND, Caldwell E, et al. Acute respiratory distress syndrome: the Berlin Definition. JAMA. 2012;307(23):2526-33. doi: 10.1001/jama.2012.5669. [PubMed: 22797452].

14. Baid H. Patient Safety: Identifying and Managing Complications of Mechanical Ventilation. Crit Care Nurs Clin North Am. 2016;28(4):45162. doi: 10.1016/j.cnc.2016.07.005. [PubMed: 28236392].

15. Nemer SN, Barbas CS, Caldeira JB, Carias TC, Santos RG, Almeida LC, et al. A new integrative weaning index of discontinuation from mechanical ventilation. Crit Care. 2009;13(5):R152. doi: 10.1186/cc8051. [PubMed: 19772625]. [PubMed Central: PMC2784374].

16. Epstein SK, Parsons P. Weaning from mechanical ventilation: readiness testing. Walthman (MA): UpToDate; 2013. Available from: https://www.uptodate.com/contents/weaning-from-mechanicalventilation-readiness-testing.

17. Arnal JM, Garnero A, Saoli M, Chatburn RL. Parameters for Simulation of Adult Subjects During Mechanical Ventilation. Respir Care. 2018;63(2):158-68. doi: 10.4187/respcare.05775. [PubMed: 29042486].

18. Desai JP, Moustarah F. Pulmonary Compliance. Treasure Island (FL): StatPearls; 2021.

19. Das N, Verstraete K, Topalovic M, Aerts J, Janssens W. Estimating Airway Resistance from Forced Expiration in Spirometry. Appl Sci. 2019;9(14). doi: 10.3390/app9142842.

20. Rahimzadeh P, Faiz HR, Farahmandrad R, Hassanlouei B, Habibi A, Hedayati Emami S, et al. Clinical Features and Prognosis of Invasive Ventilation in Hospitalized Patients with COVID-19: A Retrospective Study. Anesth Pain Med. 2020;10(6). e108773. doi: 10.5812/aapm.108773. [PubMed: 34150575]. [PubMed Central: PMC8207877].

21. Rahimzadeh P, Amniati S, Farahmandrad R, Faiz SHR, Hedayati Emami S, Habibi A. Clinical Characteristics of Critically Ill Patients Infected with COVID-19 in Rasoul Akram Hospital in Iran: A Single Center Study. Anesth Pain Med. 2020;10(5). e107211. doi: 10.5812/aapm.107211. [PubMed: 34150569]. [PubMed Central: PMC8207836].

22. Sanaie S, Hosseini MS, Karrubi F, Iranpour A, Mahmoodpoor A. Impact of Body Mass Index on the Mortality of Critically Ill Patients Admitted to the Intensive Care Unit: An Observational Study. Anesth Pain Med. 2021;11(1). e108561. doi: 10.5812/aapm.108561. [PubMed: 34249664]. [PubMed Central: PMC8256440].

23. Mahmoodpoor A, Shadvar K, Ghamari AA, Mohammadzadeh Lameh M, Asghari Ardebili R, Hamidi M, et al. Management of Critically Ill Patients with COVID-19: What We Learned and What We Do. Anesth Pain Med. 2020;10(3). e104900. doi: 10.5812/aapm.104900. [PubMed: 32944565]. [PubMed Central: PMC7472789].

24. Schultz K, D'Aquino LC, Soares MR, Gimenez A, Pereira CA. Lung volumes and airway resistance in patients with a possible restrictive pattern on spirometry. J Bras Pneumol. 2016;42(5):341-7. doi: 10.1590/S1806-37562016000000091. [PubMed: 27812633]. [PubMed Central: PMC5094870].

25. Kafan S, Tadbir Vajargah K, Sheikhvatan M, Tabrizi G, Salimzadeh A,
Montazeri M, et al. Predicting Risk Score for Mechanical Ventilation in Hospitalized Adult Patients Suffering from COVID-19. Anesth Pain Med.2021;11(2). e112424. doi:10.5812/aapm.112424. [PubMed:34336617] [PubMed Central: PMC8314086].

26. Ahmadi A, Foroghi Ghomi SY, Lotfi S. Controlled Modes Can Be as Effective as CPAP and BiPAP in Non-invasive Ventilation in COVID-19. Anesth Pain Med. 2021;11(5). e120405. doi: 10.5812/aapm.120405.

27. Atashkhoei S, Yavari N, Zarrintan M, Bilejani E, Zarrintan S. Effect of Different Levels of Positive End-Expiratory Pressure (PEEP) on Respiratory Status during Gynecologic Laparoscopy. Anesth Pain Med. 2020;10(2). e100075. doi: 10.5812/aapm.100075. [PubMed: 32637348] [PubMed Central: PMC7322673].

28. Haryalchi K, Heidarzadeh A, Abedinzade M, Olangian-Tehrani S, Ghaz anfar Tehran S. The Importance of Happy Hypoxemia in COVID-19. Anesth Pain Med. 2021;11(1). e111872. doi: 10.5812/aapm.111872. [PubMed: 34221943]. [PubMed Central: PMC8239260].

29. U RA, Verma K. Happy Hypoxemia in COVID-19-A Neural Hypothesis. ACS Chem Neurosci. 2020;11(13):1865-7. doi: 10.1021/acschemneuro.0c00318. [PubMed: 32530597].

30. Asri S, Hosseinzadeh H, Eydi M, Marahem M, Dehghani A, Soleimanpour H. Effect of Dexmedetomidine Combined with Inhalation of Isoflurane on Oxygenation Following One-Lung Ventilation in Thoracic Surgery. Anesth Pain Med. 2020;10(1). e95287. doi: 10.5812/aapm.95287. [PubMed: 32309196]. [PubMed Central: PMC7145426].

31. Yousefian M, Sadegi SRGP, Sakaki M. Vitamin D supplements' effect on expediting the weaning process in patients with the stroke. Electron J Gen Med. 2019;16(2). doi: 10.29333/ejgm/94224.

32. Sanaie S, Mahmoodpoor A, Hamishehkar H, Shadvar K, Salimi N, Montazer M, et al. Association Between Disease Severity and Calcium Concentration in Critically Ill Patients Admitted to Intensive Care Unit. Anesth Pain Med. 2018;8(1). e57583. doi: 10.5812/aapm.57583. [PubMed: 29868455]. [PubMed Central: PMC5970362].

33. Okabe Y, Asaga T, Bekku S, Suzuki H, Kanda K, Yoda T, et al. Lung-thorax compliance measured during a spontaneous breathing trial is a good index of extubation failure in the surgical intensive care unit: a retrospective cohort study. J Intensive Care. 2018;6:44. doi: 10.1186/s40560018-0313-9. [PubMed: 30083347]. [PubMed Central: PMC6069862].

34. Candik P, Rybar D, Depta F, Sabol F, Kolesar A, Galkova K, et al. Relationship between dynamic expiratory time constant tau(edyn) and parameters of breathing cycle in pressure support ventilation mode. Physiol Res. 2018;67(6):875-9. doi: 10.33549/physiolres.933750. [PubMed: 30204464].

35. Basiri R, Ghelichli M, M Lari S, Vahidi Rad A. The Critical-Care Pain Observation Tool: A useful tool for pain assessment in intensive care units. J Cardio-Thoracic Med. 2018;6(3):329-31.

36. Mohammadi M, Khafaee Pour Khamseh A, Varpaei HA. Invasive Airway "Intubation" in COVID-19 Patients; Statistics, Causes, and Recommendations: A Review Article. Anesth Pain Med. 2021;11(3). e115868. doi: 10.5812/aapm.115868. [PubMed: 34540642]. [PubMed Central: PMC8438719].

37. Al-Rawas N, Banner MJ, Euliano NR, Tams CG, Brown J, Martin AD, et al. Expiratory time constant for determinations of plateau pressure, respiratory system compliance, and total resistance. Crit Care. 2013;17(1):R23. doi: 10.1186/cc12500. [PubMed: 23384402]. [PubMed Central: PMC4056774]. 